\title{
Potential lifetime quality of life benefits of choroideremia gene therapy: projections from a clinically informed decision model
}

\author{
Celine-Lea Halioua-Haubold ${ }^{1}$ Jasleen K. Jolly $\mathbb{B}^{2,3,4} \cdot$ James A. Smith $\mathbb{D}^{5,6} \cdot$ Rafael Pinedo-Villanueva $\mathbb{B}^{5}$. \\ David A. Brindley ${ }^{1,6} \cdot$ Robert E. MacLaren ${ }^{2,3,4}$
}

Received: 29 May 2018 / Revised: 13 September 2018 / Accepted: 15 March 2019 / Published online: 17 July 2019

(c) The Author(s), under exclusive licence to The Royal College of Ophthalmologists 2019

\section{Learning Objectives}

Upon completion of this activity, participants will be able to:

1. Describe the estimated lifetime benefit of gene therapy for the retinal dystrophy choroideremia, according to a Markov model of disease progression informed by clinical data of AAV.REP1 and voretigene neparvovec.

2. Illustrate the effects of discount rate and sensitivity analysis on estimated lifetime benefit of gene therapy for choroideremia, according to a Markov model of disease progression informed by clinical data of AAV.REP1 and voretigene neparvovec.

3. Identify clinical implications of the estimated lifetime benefit of gene therapy for choroideremia, according to a Markov model of disease progression informed by clinical data of AAV.REP1 and voretigene neparvovec.

\section{Continuing Medical Education}

In support of improving patient care, this activity has been planned and implemented by Medscape, LLC and Springer Nature.

Medscape, LLC is jointly accredited by the Accreditation Council for Continuing Medical Education (ACCME), the Accreditation Council for Pharmacy Education (ACPE), and the American Nurses Credentialing Center (ANCC), to provide continuing education for the healthcare team.

Medscape, LLC designates this Journal-based CME activity for a maximum of 1.0 AMA PRA Category 1 Credit(s) ${ }^{\mathrm{TM}}$. Physicians should claim only the credit commensurate with the extent of their participation in the activity.

\section{Credit hours}

1.0

Release date: 17 July 2019

Expiration date: 17 July 2020

Post-test link: https://medscape.org/eye/posttest913835

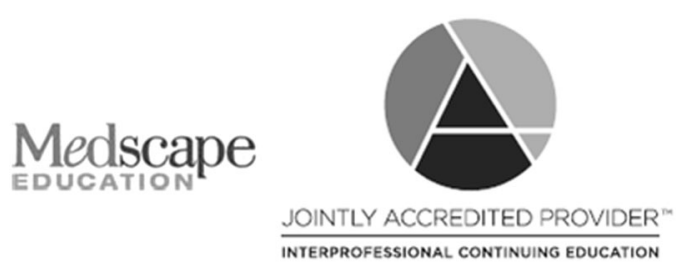

Co-senior authors: Rafael Pinedo-Villanueva, David A. Brindley, Robert E. MacLaren

Supplementary information The online version of this article (https:// doi.org/10.1038/s41433-019-0492-1) contains supplementary material, which is available to authorized users.

Rafael Pinedo-Villanueva

rafael.pinedo@ndorms.ox.ac.uk

1 Department of Paediatrics, University of Oxford, Oxford, UK

2 Nuffield Laboratory of Ophthalmology, Department of Clinical Neurosciences, University of Oxford, Oxford, UK

3 Moorfields Eye Hospital, London, UK
4 Oxford University Hospitals National Health Service Foundation Trust, Oxford, UK

5 Nuffield Departments of Orthopaedics, Rheumatology, and Musculoskeletal Sciences, University of Oxford, Oxford, UK

6 Oxford-UCL Centre for the Advancement of Sustainable Medical Innovation, University of Oxford, Oxford, UK 


\section{Authors/Editor disclosure information}

Sobha Sivaprasad has disclosed the following relevant financial relationships: Served as an advisor or consultant for Allergan, Bayer, Boehringer Ingelheim, Roche, Heidelberg, Optos. Served as a speaker or a member of a speakers bureau for Bayer, Allergan, Novartis, Optos. Received grants for clinical research from: Bayer, Boehringer Ingelheim, Allergan, Novartis, Optos. REM has disclosed the following relevant financial relationships: Served as an advisor or consultant for Nightstar Therapeutics plc; Spark Therapeutics, Inc. Served as a speaker or a member of a speakers bureau for Nightstar Therapeutics plc. Received grants for clinical research from Nightstar Therapeutics plc. CLHH, JKJ, JAS, RPV, and DAB have disclosed no relevant financial relationships.

\section{Journal CME author disclosure information}

Laurie Barclay, MD: freelance writer and reviewer. Laurie Barclay, MD, has disclosed no relevant financial relationships.

\section{Abstract}

Background The first gene therapy for an inherited retinal dystrophy recently received market approval in the United States; multiple other gene therapies are in the clinical pipeline. Thus far, gene therapy has commanded prices in the range of $\$ 500,000$ to over $\$ 1,000,000$ for the one-time doses and have been indicated for highly orphan diseases where there is no other viable treatment option. To be adopted by healthcare systems, gene therapy will need to show clinical benefit in line with its increased costs. Before longitudinal patient studies are available, model-based estimations will be necessary to project the full clinical benefit of gene therapy.

Methods To investigate the lifetime benefit of gene therapy for the retinal dystrophy choroideremia, we have built a Markov model of disease progression informed by clinical data of AAV.REP1 and voretigene neparvovec (Luxturna, Spark Therapeutics). Gene therapy patient benefit was estimated by quality-adjusted life years (QALYs) in three hypothetical disease severity patient groups. The severity of disease was defined by the combined effect of remaining retinal area and visual acuity and assigned corresponding health utility values.

Results Early-stage patients treated with gene therapy were estimated to gain, in average, 14.30 QALYs over standard-ofcare, mid-stage patients 6.22 QALYs, and late-stage patients 1.48 QALYs over untreated patients during their lifetime owing to treatment. Cost-effectiveness was not assessed as AAV.REP1 is still in clinical trials.

Conclusions In young adults in the earlier stages of choroideremia, successful gene therapy is expected to provide a significant increase in health-related quality of life.

\section{Introduction}

Voretigene neparvovec-rzyl (Luxturna), a gene therapy product for RPE65-mediated retinitis pigmentosa, recently became one of the first market-approved gene therapy products in the United States [1]. It has been priced at $\$ 850,000$ for the onetime subretinal injection to both eyes [2]. This high cost is seen across the gene therapy field: market-approved gene therapies alipogene tiparvovec (Glybera) and Strimvelis fall within a similar price range, with Glybera priced at $\$ 1.2$ million before being removed from market and Strimvelis at $\$ 650,000$ for the one-time series of injections [3, 4].

The strength of gene therapies-their specificity and targeted mechanism of action to address the direct genetic cause of the disease-makes them an attractive treatment strategy for simple congenital disorders, and often the only treatment option available for such diseases. However, this also restricts their efficacy to populations of a certain genotype. For example, Luxturna is only applicable to $2 \%$ of the total United States retinitis pigmentosa population [5]. Within these already small numbers, the eligible populations may dwindle further for a variety of patient-specific reasons including stage of degeneration, subtype of the mutation, and previous engagement in clinical trials. In addition to high development costs and small population sizes, the mechanism of action of gene therapies adds another layer of complexity to their pricing: their full effect is often given in a single treatment session [6-8].

For each of the gene therapies highlighted here, including those for retinal dystrophies, there is no sufficient alternative treatment. Assuming gene therapy continues to prove itself in the clinic, their high prices pose a significant threat to patient accessibility. Nationalized healthcare systems, such as the United Kingdom's National Health System (NHS), often have strict caps on cost-benefit ratio for a therapy to be adopted into the system. Strimvelis was recommended for use in the NHS in October 2017 by the National Institute for Health and Care Excellence (NICE), which among other activities publishes on the costeffectiveness of new therapies for use in the context of the NHS [3]. Glybera was deemed too costly for its demonstrated patient benefit by both the German and French healthcare systems and this played a role in its eventual withdrawal from market [4]. Luxturna was recently 
Table 1 Choroideremia gene therapy clinical trials

\begin{tabular}{lllll}
\hline NCT number & Phase & Sponsor & Dates & Enrollment size \\
\hline NCT01461213* & Phase I/II & University of Oxford & $2011-2017$ & 14 \\
NCT02077361 & Phase I/II & University of Alberta & $2015-2018$ & 6 \\
NCT02341807 & Phase I/II & Spark Therapeutics Inc & $2015-2019$ & 15 \\
NCT02553135 & Phase II & University of Miami & $2015-2021$ & 6 \\
NCT02671539 & Phase II & University of Tübingen & $2016-2018$ & 6 \\
NCT02407678* & Phase II & University of Oxford & $2016-2019$ & 30 \\
In progress* & Phase III & Nightstar Therapeutics Inc & $2019-2020$ & Not yet known \\
\hline
\end{tabular}

Asterisk indictates AAV.REP1 clinical trials deemed not to be cost-effective at its price point by the United States non-profit Institute for Clinical and Economic Review [9]. Private healthcare providers in the United States have shown reluctance in reimbursing similarly expensive orphan therapies, such as Sarepta Therapeutics' Duchenne's muscular dystrophy drug eteplirsen (Exondys 51) [10]. To be potentially adopted by healthcare systems, gene therapies will at minimum need to prove their capacity to deliver patient benefit at scale with their high costs.

The congenital retinal dystrophy choroideremia is characteristic of a gene therapy target: it is an orphan disease, monogenic, has no effective treatment, and has a severe predictable impact on the patient's quality of life. Choroideremia is caused by an X-linked loss-of-function mutation of Rab escort protein 1 (REP1) in the retinal pigment epithelium, resulting in progressive vision loss and eventual severe vision impairment in most of the affected around age 40 [11]. Multiple clinical trials have been conducted for choroideremia gene therapy, and one candidate will soon enter Phase III clinical trials (Table 1). Spark Therapeutics Inc and Nightstar Therapeutics Inc are developing adeno-associated virus (AAV)-based therapies for choroideremia, which deliver a functional copy of the REP1 gene (AAV.REP1).

To investigate the potential patient benefit of gene therapy on a population amenable to this treatment strategy, we conducted a case study of choroideremia gene therapy. We built a Markov model of choroideremia disease progression and populated it with clinical data from AAV.REP1, Luxturna, and published natural history studies. The health impact of choroideremia-associated visual function loss over time was assessed using quality-adjusted life years (QALYs), a measure of the impact of a disease on a patient's quality of life often used for decision making in healthcare. Using this model, we assessed the patient benefit provided by a curative gene therapy (defined by arresting progression or slowing it down to a rate that is no longer clinically significant) at various stages of the disease progression. Choroideremia gene therapy can act as a case study for the potential patient benefit, and more broadly the potential clinical viability, of retinal gene therapy.

\section{Methods}

\section{Overview}

A simplified schematic of the model is shown in Fig. 1 (see eTables 1-4, eFigs 1-4, and supplementary methods for detailed model design, programming, equations, and explanation). The combined effect of remaining retinal area and visual acuity was categorized into five health states with corresponding health utility values: "healthy", "hindered", "functionally blind", "blind", and "death". The patient cohort progressed through the health states according to assigned transition probabilities, using a cycle length of 1year, accumulating QALYs from point of entry in the model (treatment decision) until death. Three representative, hypothetical choroideremia patient subgroups were defined: 20 -year-old patients with no significant retinal area and normal visual acuity (referred to here as the early-stage cohort); 45-year-old patients with significant retinal area loss but no visual acuity deficit (mid-stage cohort); and 60year old patients with both significant retinal area and visual acuity loss (late-stage cohort). The model was built to simulate the full lifetime of patients from point of treatment decision (untreated or gene therapy), as the disease as well as the therapy would impact their quality of life for their entire lives. The gene therapy was assumed to be $93 \%$ successful, with success defined as halting retinal area degeneration and the subretinal injection proceeding without any serious adverse events. The gene therapy was assumed to not rescue visual acuity or retinal area, only to stop the progression of the disease. The model was built using the software TreeAge Pro (TreeAge Software, Williamstown, Massachusetts: TreeAge Software treeage.com).

Model input parameters relating to the gene therapy were obtained from the published results as of late 2017 of the Phase I/II clinical trials for choroideremia, natural history studies, and Luxturna [12-14]. Luxturna is indicated for a different retinal degenerative disorder but has a broadly similar mechanism of action. Quality of life was only considered after treatment decision. 


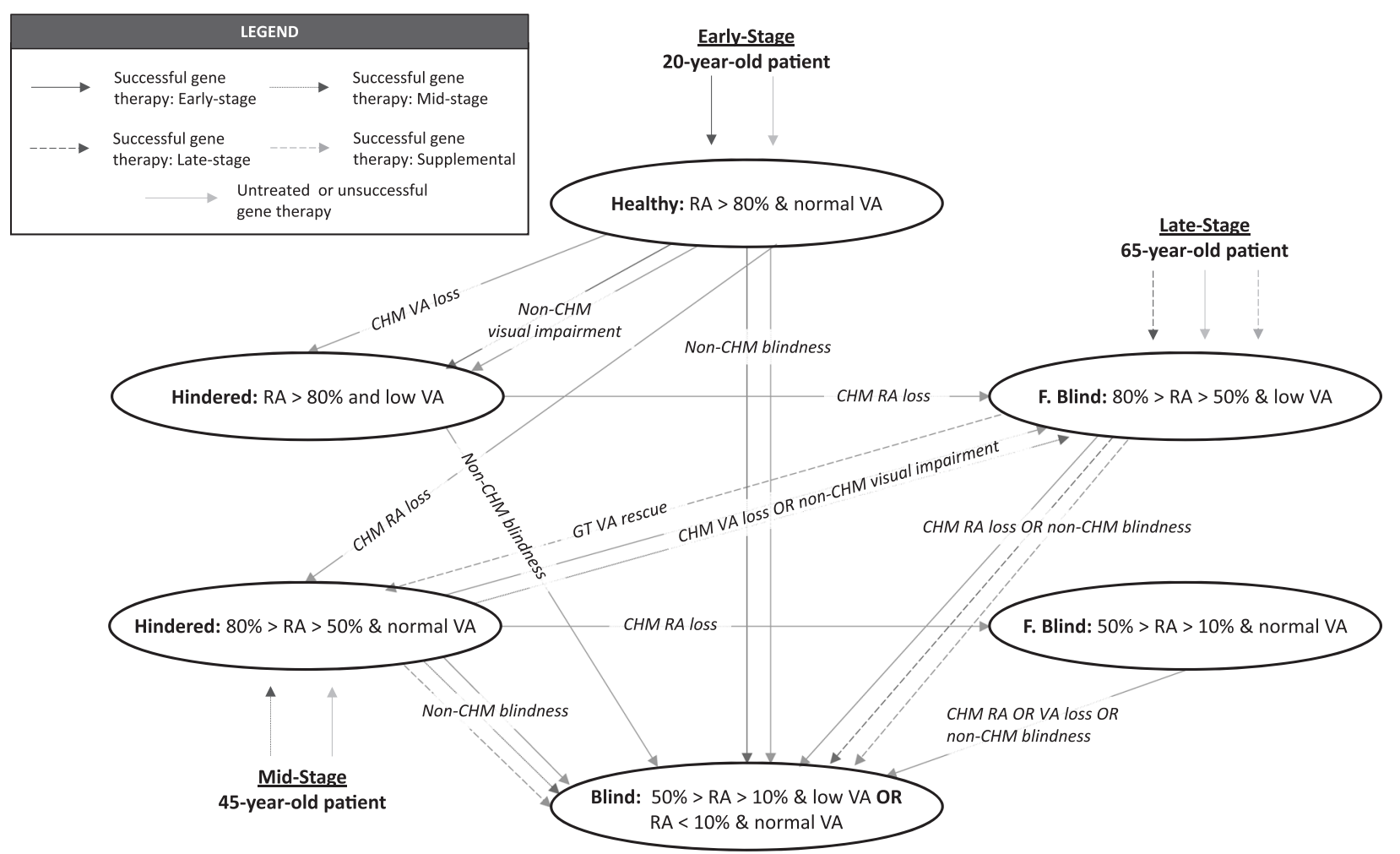

Fig. 1 Overview of Choroideremia Markov Model. The model incorporated both the progressive loss of retinal area (RA), owing to photoreceptor degeneration, and visual acuity (VA) loss owing to choroideremia or common non-choroideremia causes

\section{Visual function}

The model assessed the progression of choroideremiaassociated visual impairment in untreated and gene therapy patients. Degree of impairment was defined by a combination of loss of visual acuity and visual field, the latter estimated by retinal area. As is expected from clinical data, the gene therapy did not restore already-lost retinal area. Retinal area loss in choroideremia has been well defined and follows an exponential decay pattern with advancing age [15].

Gene therapy and untreated patients still develop nonchoroideremia-associated visual impairment or blindness for causes including glaucoma, injury, and diabetic retinopathy. Hence, to include the effects of these more-common conditions, the probability of non-choroideremia visual impairment or blindness was also calculated, using the United States National Eye Institute Data on the prevalence of low vision and blindness by age group [16].

\section{Intervention}

The gene therapy was assumed to slow retinal area loss to the point where it would no longer be clinically significant. That is to say that the patients would still retain useful vision until they died from other causes. The efficacy of the gene therapy was assumed to be $93 \%$, the demonstrated success rate of Luxturna in Phase III trials in a cohort of 29 [17].

\section{Health utility}

Patient benefit was estimated using QALYs, a measure combining survival and health-related quality of life where a value of 1 represents a full year lived in perfect health. The authors are unaware of previously published assessments of the impact of choroideremia on patient quality of life. Therefore, the health state utility values used in the model were informed by previous assessments of the impact of non-choroideremia-associated visual function loss on patient quality of life $[18,19]$. Hence, the "healthy", "hindered", "functionally blind", and "blind" health states were assumed to have health utility values of $0.870,0.715,0.600$, and 0.390 QALYs, respectively, with "death" having by definition a utility of 0 QALYs. QALYs accumulated for each year in the corresponding state were therefore equal to such health utility values.

\section{Patient benefit}

As we used a cohort model, the mean QALY outcome accounts for the $7 \%$ failure rate of the gene therapy, the incidence of non-choroideremia-associated visual 
impairment and blindness, and varying lifespans of treated patients. An individual patient with successful gene therapy who lives a long life would benefit more from gene therapy than the reported mean for the cohort, whereas a patient who lives a shorter life or develops non-choroideremia visual impairment would accrue less benefit than the reported mean over their lifetime. Cohort models are useful for a healthcare system context, where the average expected population health gains can be compared against alternatives.

\section{Late responders}

Preliminary AAV.REP1 clinical data has suggested that the gene therapy may not only halt retinal degeneration, but also improve visual acuity in patients where this has decreased owing to choroideremia disease progression [20]. Owing to the small sample size suggesting this gene therapy activity, the authors did not believe it was sufficient to include visual acuity restoration in the primary model; therefore, patient cohorts were run under the assumption that gene therapy can only halt retinal degeneration. To explore the impact of potential visual acuity restoration, a hypothetical supplemental patient group was put through the model: a 60 -year old patient with between $50 \%$ and $80 \%$ remaining retinal area and visual acuity loss. The gene therapy, if successful, was assumed to have a $95 \%, 85 \%$, or $65 \%$ probability of restoring visual acuity in addition to halting retinal area degeneration.

\section{Discounting}

There is a time preference to health outcomes: society and patients prefer benefits from their investment sooner rather than later [21]. Discounting is commonly used to reflect this in quantified outcomes. This model did not use a discount rate as costs were not considered, however a sensitivity analysis was run with a discount rate of $1.5 \%$ and $3.0 \% ; 3.0 \%$ is the standard discount rate used by NICE, with $1.5 \%$ used for some subsets of long-term interventions [22].

\section{Sensitivity analyses}

To determine the impact of the chosen health utility values on the outcomes, multiple sensitivity analyses were conducted. One-way sensitivity analysis was conducted using $\pm 15 \%$ of the chosen health utility values for each patient cohort; that is to say, only one health utility value was varied at a time and all other parameters were kept at their base case values. An additional sensitivity analysis was run where all health utility values were varied $-15 \%$ or $+15 \%$ from the utilized value.

\section{Results}

\section{Patient outcomes}

Patients who did not receive gene therapy remained untreated and steadily lost retinal area and visual acuity over their lifetime, as is expected owing to choroideremia disease progression. Gene therapy largely prevented this in the treated cohort (Fig. 2). As expected, treatment at a younger age and less-severe health state netted the largest patient benefit as estimated by QALYs.

The lifetime QALYs accumulated between gene therapy versus untreated patients were compared for each hypothetical patient (Table 2 and Fig. 3). For a 20 -year-old who is treated before significant retinal area and visual acuity loss (early-stage), gene therapy would provide an extra 14.30 QALYs over the patients' lifetime after point of treatment when compared with the untreated patient. The 45-year-old patient with retinal area loss but healthy visual acuity (mid-stage) would gain an extra 6.22 QALYs from the gene therapy over not being treated. The latest stage patient, 60-years-old with significant retinal area and visual acuity degeneration (late-stage), would still benefit from the treatment, expecting to gain an additional 1.48 QALYs.

\section{Late responder group outcomes}

Preliminary clinical data with a small sample showed visual acuity restoration in all treated late-stage patients with previous visual acuity loss [20]. The cumulative QALY benefit in the $95 \%, 65 \%$, and $50 \%$ groups was 3.66, 2.97, and 2.63 QALYs, respectively. The benefit without visual acuity restoration probability incorporated was 1.48 QALYs (eFig. 5).

\section{Discount rate}

The QALY outcomes showed noted sensitivity to the discount rate (Table 2, eTable 5). The base model utilized no discount rate. A sensitivity analysis was run with a $1.5 \%$ and $3 \%$ annual discount rate on the health benefits. At a $1.5 \%$ discount rate, the expected cumulative benefit for early-, mid-, and late-stage patients was 8.65, 4.46, and 1.14 QALYs, respectively. At a 3\% discount rate, these values lowered significantly to 5.66, 3.31, and 0.90 QALYs, respectively.

\section{Sensitivity analysis of health state utility values}

Two methods of sensitivity analyses were applied to the health utility values (eTables 6 and 7). One-way sensitivity analysis showed the early-stage cohort outcomes were most sensitive to utility value variances of "healthy": gene therapy 

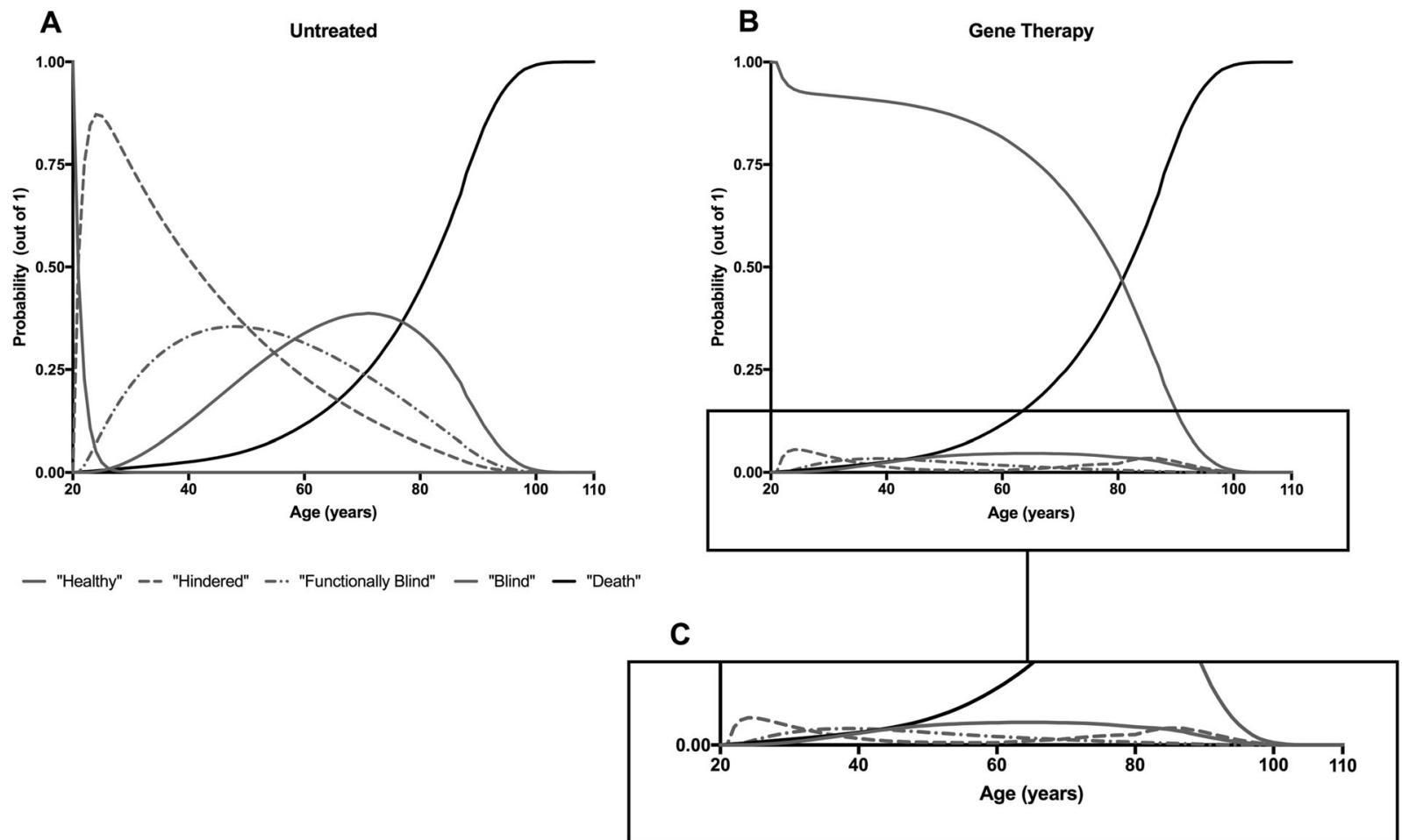

Fig. 2 Probability of health state progression by age in gene therapy and untreated patients. a Untreated patients progressed through worsening health states quickly, with approximately half of the cohort having significantly impaired vision by age 65. b Gene therapy patients largely did not progress past "healthy" over their lifetime.

Table 2 Quality-adjusted life years gained by gene therapy

\begin{tabular}{cccc}
\hline Patient group & Gene therapy & Standard-of-care & Incremental \\
\hline No discount rate & & & \\
Early stage & 49.49 QALYs & 35.19 QALYs & 14.30 QALYs \\
Mid stage & 24.71 QALYs & 18.49 QALYs & 6.22 QALYs \\
Late stage & 13.48 QALYs & 12.00 QALYs & 1.48 QALYs \\
1.5\% discount rate & & & \\
Early stage & 32.74 QALYs & 24.09 QALYs & 8.65 QALYs \\
Mid stage & 18.93 QALYs & 14.47 QALYs & 4.46 QALYs \\
Late stage & 11.24 QALYs & 10.10 QALYs & 1.14 QALYs \\
\hline
\end{tabular}

The benefit of the gene therapy was quantified using quality-adjusted life years (QALYs) and compared with the expected QALY gain in an untreated patient of the same age

benefit varied from 7.52 to 21.06 QALYs using the lower and higher health utility value respectively (with 14.33 QALYs being the base case model outcome). The mid- and late-stage followed similar patterns: varying the utility value for "hindered" gave values of incremental benefit of gene therapy over no treatment of 3.14-9.30 QALYs (6.22 QALYs unvaried) and varying the utility value for "functionally blind" gave a range of 1.90-1.07 (1.48 QALYs). c Zoomed in portion of the gene therapy cohort. A small subset of the group continued to have disease progression due to failure of the gene therapy. An increase in hindered patients and small increase in blind patients is seen at $\sim 85$ years, owing to the increased incidence of nonchoroideremia-associated visual function loss and blindness

In addition, versions of the model were run where all the health utility values were either lowered $15 \%$ or raised $15 \%$. This did not notably vary the gene therapy benefit.

\section{Sensitivity analysis of gene therapy success rate}

The model showed little sensitivity to variances of the success rate in all patient cohorts. The model utilized a gene therapy success rate of $93 \%$, with success defined as the gene therapy having its full therapeutic effect on at least one eye. Sensitivity analyses were run with success rates of $100 \%, 88.5 \%$, and $83.5 \%$ (eTable 8). Lower rates were not investigated due to the clinical data supporting a high $(>90 \%)$ success rate.

\section{Discussion}

In the ideal patient treatment scenario-treating as soon as diagnosed, before significant disease progression-choroideremia gene therapy has a large positive impact on the quality of life of early-stage patients for the remainder of their life. Strictly evaluating this patient category, there are strong quality of life arguments for providing choroideremia gene 

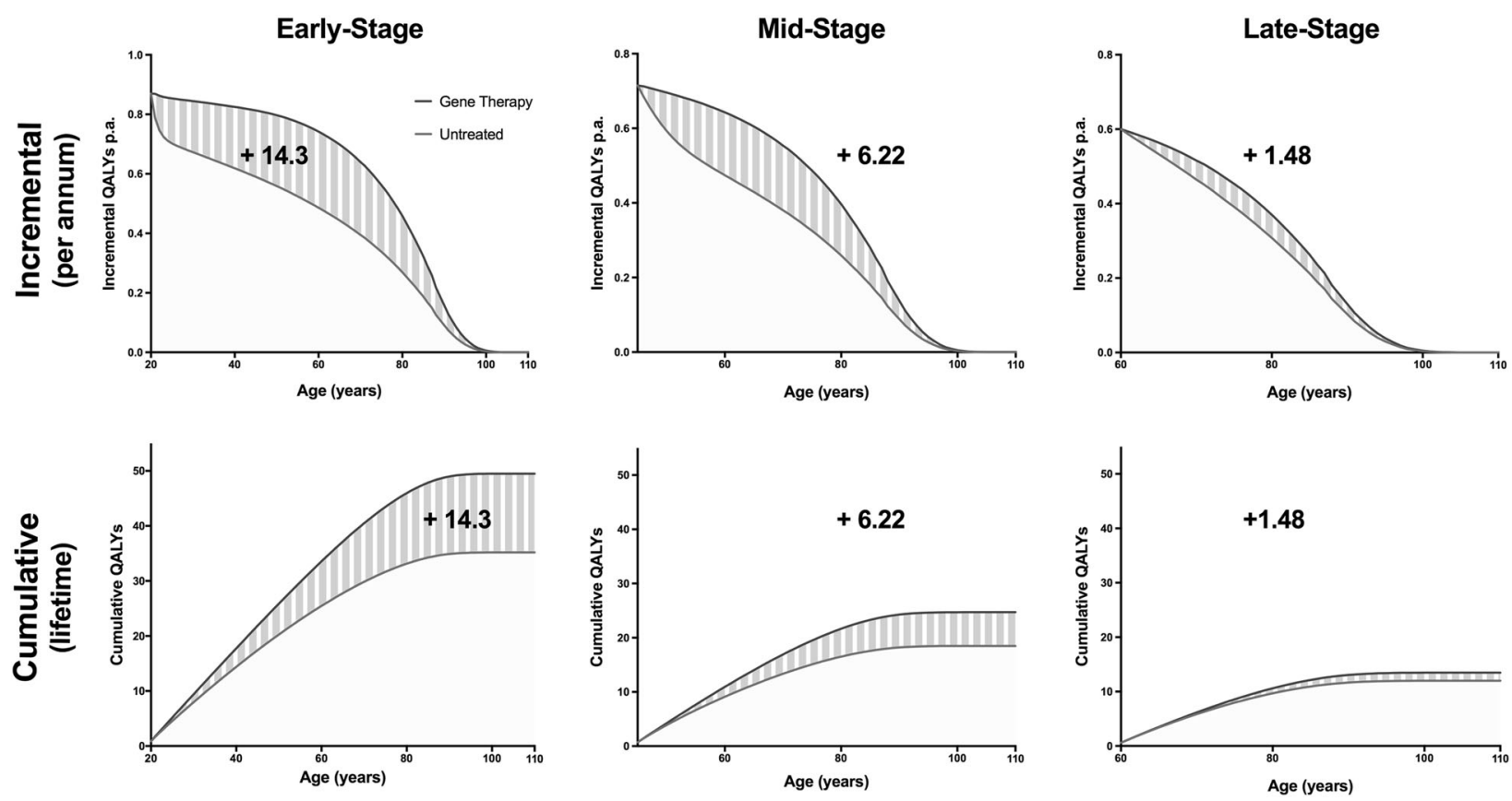

Fig. 3 Cumulative and incremental benefit of gene therapy in three patient populations. Incremental (per year) and cumulative (lifetime)

quality of life in choroideremia patients. The benefit of gene therapy over untreated patients was largely accrued later in life

therapy to every early-stage patient. As expected, the patient benefit of gene therapy decreases the further degenerated the patient's vision is at point of treatment.

As there is currently no treatment for choroideremia, there is a distribution of choroideremia patients across all levels of the degeneration spectrum. For the more-advanced patients, the economic argument for providing an expensive therapy may be more challenging from a strictly costeffectiveness perspective. Our brief exploration of treating these advanced patients demonstrated the significantly smaller QALY gain provided by the gene therapy over no treatment. Clinical trial data are needed to determine the realized likelihood of AAV.REP1 improving visual acuity -if this is the case, then the benefit of the gene therapy for late-stage patients may be much larger than what our preliminary model shows. Even with this boost, however, gene therapy may still not be cost-effective for such patient populations.

It is not the aim of this study to justify the developers of gene therapies to continue requiring such high prices for future gene therapy products; indeed, as more gene therapies enter the market, their costs should decline as the manufacturing and development strategy for therapies utilizing similar strategies (for example, AAV-based therapies) becomes standardized and streamlined. However, gene therapy will likely remain at a higher cost than small molecule or protein therapeutics for the foreseeable future owing in part to their complex components and high degree of specificity for certain small patient populations. As such, developers of gene therapies should be held to high standards on efficacy. Unlike in small molecule drugs, where a moderate improvement in patient outcomes may justify clinical adoption presuming it is safe and needed, there will likely be little tolerance by healthcare providers to reimburse at current gene therapy prices. Ultimately, it will be essential to give the treatment early on in life to prevent sight loss; this is also likely to be the period during which the gene therapy treatment is the most cost-effective.

This model provides one of the first academic insights into the potential lifetime patient benefit of retinal gene therapy based on clinical data, and the first of choroideremia gene therapy. It is also unique in that it evaluates a candidate therapy before it has received market approval, conversely to the standard practice of evaluating at point of market entry. As more gene therapies enter clinical trials and the market, models such as these can provide insight into the sustainability of their clinical use before long-term patient outcomes are available. In addition, models such as these can provide valuable insight into gene therapy development strategy. As molecular tools widen the indications, which are possible to address with gene therapy, modeling can assist researchers and industry in identifying the most amenable diseases for these costly therapeutic strategies and prioritizing the therapy development pipeline. As was seen with Glybera, which showed some efficacy but in the end was not clinically viable owing to its price point, it is crucial that, in the development of extraordinary therapies, the adoption climate in which they will arrive is kept in mind. 


\section{Limitations}

A significant limitation of this model-and likely any model of an orphan disease therapy-was the small sample sizes in the patient data. We have conducted multiple sensitivity analyses to address uncertainties in the results presented deriving from data variability. Usually, cost-effectiveness analyses inform models with transition probabilities derived from large clinical data sets on market-approved therapeutics. Clinical trials for choroideremia gene therapies have to date had trial sizes of only up to $\sim 20$ patients. Also for this reason, the transition probabilities within the model did not incorporate confidence intervals and as such our results carry with them a higher degree of uncertainty. As no choroideremia gene therapy has not completed Phase III clinical trials, the gene therapy success rate was assumed from published earlier-phase clinical trial data and from Luxturna's Phase III study. Another consequence of choroideremia's low incidence was the lack of health utility values for the disease. As the authors are not aware of published choroideremia-specific health utility values of a representative sample, utility values for nonchoroideremia visual impairment and blindness were used. Owing to the primary role of health utility values in cost-effectiveness models, assessments of health utility for orphan indications may prove crucial in facilitating accurate reimbursement decisions by healthcare providers.

\section{Summary}

\section{What was known before}

- Gene therapy is a good therapeutic strategy for some diseases, including monogenic retinal diseases, however it is very expensive. For a therapy to be cost-effective, it must deliver large patient benefit.

\section{What this study adds}

- An estimate of the lifetime benefit of gene therapy in choroideremia. Preliminary analysis of the time to benefit of gene therapy.

Acknowledgements CLHH thanks the SENS Research Foundation for their continued intellectual support. CLHH is funded by the NIHR. JKJ is funded by the National Institute for Health Research (NIHR). JS is funded primarily by an MRC UK DPhil Studentship. DB gratefully acknowledges personal funding from the Oxford Musculoskeletal National Institute for Health Research Biomedical Research Unit (NIHR BRU), the Saïd Foundation and the SENS Research
Foundation. REM receives funding from the Oxford University Hospitals NHS Foundation Trust NIHR Biomedical Research Centre (BRC). CLHH has been a consultant for multiple life sciences companies and biotechnology investment firms. DB is a stockholder in Translation Ventures Ltd. (Charlbury, UK) and IP Asset Ventures Ltd. and Biolacuna Ltd., companies that among other services provide biomanufacturing, regulatory, and financial advice to pharmaceutical clients. DB also is subject to the CFA Institute's codes, standards, and guidelines, so he must stress that this piece is provided for academic interest only and must not be construed in any way as an investment recommendation. In addition, at time of publication, DB and the organizations with which he is affiliated may or may not have agreed to and/or pending funding commitments from the organizations named herein. REM is a Founder of Nightstar Therapeutics Inc and a consultant to Spark Therapeutics Inc. REM is acting as an independent academic and neither Nightstar nor Spark had any role in the conception or execution of this research study. CLHH is not funded by Nightstar Therapeutics, Spark Therapeutics, or any other entity with commercial interest in gene therapy.

Author contributions CLHH: developed model and wrote manuscript. JKJ: choroideremia expertize. JS: reviewed for clarity and accessibility to a diverse audience. RPV: supervised and validated model development. DAB: copyediting. REM: choroideremia expertize and general academic support.

\section{Compliance with ethical standards}

Conflict of interest REM is a Founder of Nightstar Ltd and a consultant to Spark Therapeutics Inc. REM is acting as an independent academic and neither Nightstar nor Spark had any role in the conception or execution of this research study. CLHH does not receive funding or other incentives from NightStar, Spark Therapeutics, or any other entity with commercial interests in Choroideremia gene therapy. JKJ, JAS, RPV and DAB have no conflicts.

Publisher's note: Springer Nature remains neutral with regard to jurisdictional claims in published maps and institutional affiliations.

\section{References}

1. U.S. Food and Drug Administration. Press Announcements - FDA approves novel gene therapy to treat patients with a rare form of inherited vision loss. https://www.fda.gov/NewsEvents/New sroom/PressAnnouncements/ucm589467.htm. Accessed 5 May 2018.

2. Sangonowsky E Spark sets off gene therapy debate with $\$ 850 \mathrm{~K}$ sticker on Luxturna I FiercePharma. FiercePharma. /pharma/ spark-prices-gene-therapy-luxturna-at-850k-grabbing-top-spotpharma-s-costliest-drugs. Published 3 January 3, 2018. Accessed 25 January 2018.

3. NICE. Evaluation Consultation Document: Strimvelis for Treating Adenosine Deaminase Deficiency-Severe Combined Immunodeficiency.; 2017. https://www.nice.org.uk/guidance/GID-HST10005/ documents/evaluation-consultation-document. Accessed October 25, 2017.

4. Touchot N, Flume M. Early Insights from Commercialization of Gene Therapies in Europe. Genes. 2017;8:78.

5. SEC Filings I Investors \& Media I Spark Therapeutics. http://ir.spa rktx.com/phoenix.zhtml? $\mathrm{c}=253900 \& \mathrm{p}=$ irol-SECText $\&$ TEXT $=\mathrm{a}$ HR0cDovL2FwaS50ZW5rd216YXJkLmNvbS9ma WxpbmcueG1sP2lwYWdlPTEwMTcw NDk3JkRTRVE9MSZTRVE9MTQmU1FERVNDPVNFQ1RJ 
T05fUEFHRSZleHA9JnN1YnNpZD01Nw\%3D\%3D. Accessed 25 October 2017.

6. EMA. Glybera. Annex I. Summary of Product Characteristics. http://www.ema.europa.eu/docs/en_GB/document_library/ EPAR_-_Product_Information/human/002145/WC500135472. pdf. Accessed 30 October 2017.

7. EMA. Strimvelis. Annex I. Summary of Product Characteristics. http://www.ema.europa.eu/docs/en_GB/document_library/ EPAR_-_Product_Information/human/003854/WC500208199. pdf. Accessed 30 October 2017.

8. U.S. Food and Drug Administration. Package Insert - LUXTURNA (Voretigene Neparvovec-Rzyl).; 2017. https://www.fda.gov/dow nloads/BiologicsBloodVaccines/CellularGeneTherapyProducts/ ApprovedProducts/UCM589541.pdf. Accessed 5 May 2018.

9. Lam BL, Russell SR. Voretigene Neparvovec for Biallelic RPE65-Mediated Retinal Disease: effectiveness and value, 2007. https://icer-review.org/wp-content/uploads/2017/06/MWCEPAC_ VORETIGENE_DRAFT_EVIDENCE_REPORT_11152017.pdf.

10. Thomas K. Insurers Battle Families Over Costly Drug for Fatal Disease-The New York Times. The New York Times. https://www.nytimes.com/2017/06/22/health/duchenne-musculardystrophy-drug-exondys-51.html?smprod=nytcore-ipad\&smid $=$ nytcore-ipad-share. Published 22 June 2017. Accessed 27 October 2017.

11. van den Hurk JA, Schwartz M, van Bokhoven H, et al. Molecular basis of choroideremia (CHM): mutations involving the Rab escort protein-1 (REP-1) gene. Hum Mutat. 1997;9:110-7.

12. MacLaren RE, Groppe M, Barnard AR, et al. Retinal gene therapy in patients with choroideremia: initial findings from a phase $1 / 2$ clinical trial. The Lancet. 2014;383:1129-37.

13. Jolly JK, Xue K, Edwards TL, Groppe M, MacLaren RE. Characterizing the natural history of visual function in choroideremia using microperimetry and multimodal retinal imaging. Invest Ophthalmol Vis Sci. 2017;58:5575-83.

14. Dimopoulos IS, Hoang SC, Radziwon A, et al. Two-year results after AAV2-mediated gene therapy for choroideremia: the alberta experience. Am J Ophthalmol. 2018;193:130-42.

15. Jolly JK, Edwards TL, Moules J, Groppe M, Downes SM, MacLaren RE. A qualitative and quantitative assessment of fundus autofluorescence patterns in patients with choroideremia quantification of autofluorescence in choroideremia. Invest Ophthalmol Vis Sci. 2016;57:4498-503.

16. Low Vision Tables I National Eye Institute. https://www.nei.nih. gov/eyedata/lowvision/tables. Accessed 2 November 2017.

17. Spark Therapeutics Announces New Positive Data from Continuation of Phase 3 Trial of Voretigene Neparvovec. Spark Therapeutics Inc. - IR Site. https://www.news-releases/news-relea se-details/spark-therapeutics-announces-new-positive-datacontinuation. Accessed 5 May 2018.

18. Spark Therapeutics' Luxturna advisory committee vote sets gene therapy landmark | FierceBiotech. https://www.biotech/sparktherapeutics-luxturna-adcomm-vote-sets-gene-therapy-landmark. Accessed 25 October 2017

19. Brown GC. Vision and quality-of-life. Trans Am Ophthalmol Soc. 1999;97:473-511.

20. Edwards T, Jolly JK, Groppe M, et al. Visual acuity after retinal gene therapy for choroideremia. N Engl J Med. 2016;374:1995-6.

21. Severens JL, Milne RJ. Discounting health outcomes in economic evaluation: the ongoing debate. Value Health. 2004;7:397-401.

22. National Institute for Health and Clinical Excellence. Guide to the Methods of Technology Appraisal. London: NICE; 2013. https://www.nice.org.uk/guidance/pmg9/resources/guide-to-themethods-of-technology-appraisal-2013-pdf-2007975843781. Accessed 6 December 2017. 